\title{
Role of Plant Growth Promoters on Sugarcane Production Propagated Through Budchips in Semiarid Region of Pakistan
}

\author{
Abdul Rehman'1, Rafi Qamar1*, ${ }^{1}$ Muhammad E. Safdar, ${ }^{2}$ Atique-ur-Rehman, ${ }^{2}$ Shakeel Ahmad, \\ ${ }^{1}$ Muhammad A. Nadeem, ${ }^{3}$ Mahmood-ul-Hassan \\ 1 Department of Agronomy, College of Agriculture, University of Sargodha, Pakistan. \\ 2 Department of Agronomy, Bahauddin Zakariya University, Multan, Pakistan. \\ ${ }^{3}$ Sugarcane Research Institute, Ayub Agricultural Research Institute, Faisalabad, Pakistan.
}

\section{A R T I C L E I N F O}

\section{Article History}

Received: October 12, 2021

Revised: December 19, 2021

Accepted: December 26, 2021

\section{Keywords}

Sugarcane budchip

Growth promoters

Commercial cane sugar

Growth traits

Soaking

\section{A B S T R A C T}

One of the alternative ways of sugarcane sett planting is to sow excised sugarcane buds, popularly called as budchips. Despite the advantages of budchip technology, sugarcane growers are failed to achived higher production under field conditions due to rapid exhaustion of small sized storage tissue (budchip). Evaluate the role of varied concentrations of nephtahline acetic acid and calcium chloride on growth, production and quality of budships in semi-arid condition during two consecutive spring seasons of 2018 and 2019. Results from both years 2018 and 2019, showed that significantly $(\mathrm{P} \leq 0.05)$ improved in sprouting establishment $(86 \%)$, leaf area index $(11.98$ and 11.96), leaf area duration (1272 and 1293 days), crop growth rate (11.58 and $11.02 \mathrm{~g}$ $\mathrm{m}^{-2}$ day $\left.^{-1}\right)$, net assimilation rate (2.53 and $2.55 \mathrm{~g} \mathrm{~m}^{-2}$ day $^{-1}$ ), cane height (203 and 224 $\mathrm{cm})$, number of tillers $\mathrm{m}^{-2}$ (27 and 26), cane diameter (2.97 and $3.01 \mathrm{~cm}$ ), stripped cane yield (101.98 and $98.52 \mathrm{t} \mathrm{ha}^{-1}$ ), commercial can sugar (14.45 and 14.39\%), sugar yield (16.77 and 16.75 t ha- $^{-1}$ ) were recorded in budchips soaked with $\mathrm{CaCl} 22 \%$ + NAA $2 \mathrm{ppm}$. In above studied traits, significantly $(\mathrm{P} \leq 0.05)$ minimum was recorded in budchips soaked with $\mathrm{CaCl} 20 \%$ + NAA 0 ppm (control). On the basis of above results, it might be recommended to farmers who want to adopt this budchip technology should soaked budchips within $2 \%$ solution of $\mathrm{CaCl} 2$ with 2 ppm NAA that was suitable for improving growth, yield and quality of sugarcane budchip.

Corresponding Author: Rafi Qamar

Email: rafi.qamar@uos.edu.pk

(C) The Author(s) 2021.

\section{INTRODUCTION}

Sugarcane (Saccharum officinarum L.) is a perennial crop and conventionally propagated through vegetative part known as setts having length 25 to $30 \mathrm{~cm}$ contains 2 to 3 buds (eyes) (Patnaik et al., 2017). Generally, 6-8 tons setts on weight basis or 30,000 setts which is $10 \%$ of total cane production, is sufficient for cultivation for one hectare (Shoo, 2014). Moreover, these bulky setts create problems to the sugarcane growers in handling, transportation and storage furthermore, setts show quick deterioration that could decrease the viability of buds which ultimately leads to lower cane production (Galal, 2016). In Pakistan, sugarcane is known as a major cash crop (Khan et al., 2020) and its historical and production is presented in figure 3 (Ahmad et al., 2018).

Sugarcane budchip planting is known as new innovative planting technique, which is alternative way of conventionally sugarcane sett planting. Sugarcane budchip technology provides solution of all above problems which is associated with sett planting (Patil et al., 2018). Only 2 to 3 quintals of budchips are enough for planting one hectare which consequently save about $96 \%$ of the cane by weight 
(Jain et al., 2010). It is the most cost-effective technique for sugarcane planting and directly saves million tons of sugarcane that can be used for the preparation of sugar rather than buried in soil as seed (Patnaik et al., 2017). Instead of all these advantages of budchip technology, poor survival of budchips has noticed in farmer's field conditions (Patil et al., 2018). Survival of budchips is poor as compared to setts because of quick exhausting of water contents and food reserves (Jain et al., 2010). Therefore, to cope with this limitation in budchip technology, need to adopt an effective and proper management strategy for healthy seedling establishment from budchip. For this purpose, increase extension activity among farmer's community and design such training modules that improved the farm practices and productivity in budchip planting (Padilla-Fernandez et al., 2020).

Plant growth promotors (PGPs) are the substances which have ability in improving crop production (Iqbal et al., 2006). PGPs are being used in regulating physiological functions at low concentrations (Davies, 2010) while retarded plant growth and production at higher concentrations (Aslam et al., 2013). Naphthalene acetic acid (NAA) is an important member of synthetic auxins which is spraying at various plant growth stages for flowering initiation, restricts fruit dropping, enhances fruit setting, increases cell division and promotes root initiation (Prakash and Ganesan, 2001). Calcium is an important plant nutrient which has dominant role in the constituents of the cell wall and membrane furthermore, acts as cation and intracellular messenger in vacuole and cytosol respectively (White and Broadley, 2003). Above PGPs were applied on sugarcane budchip that significantly influence the sprouting establishment and subsequently crop growth (Rehman et al., 2021).

The study yet conduced have seen the effect of calcium and NAA on sugarcane budchips, separately. But nothing has been done to determine combined effect of calcium and NAA on sugarcane propagated through budchips, under the field conditions. The need was developed for reliable evidence about the interaction of calcium and NAA on sugarcane budchips under the agro-ecological conditions of Sargodha. Thus, study was planned to establish the interaction of various levels of calcium and NAA would affect the growth, yield and quality of sugarcane propagated through budchips.

\section{MATERIALS AND METHODS \\ Experimental site and soil}

Field experiment was executed during spring growing season of 2018 and 2019 at College of Agriculture, University of Sargodha, in district Sargodha located at $32.08^{\circ} \mathrm{N}, 72.67^{\circ} \mathrm{E}$ and $193 \mathrm{~m}$ altitude. Soil was sandy clay loam having soil bulk density $\approx 1.04 \mathrm{mg} \mathrm{m}^{-3}$ (Paull et al., 1998). Experimental field water holding and moisture ability were maintained with $33 \%$ and $70 \%$ with water, respectively. For physico-chemical analysis during 2018 and 2019, soil was air-dried and crushed than sieved out from sieve having size of $2 \mathrm{~mm}$ at $0-15 \mathrm{~cm}$ and $15-30 \mathrm{~cm}$ before experiment through adopting the methods of Ryan et al. (2001). Hydrometer techniques was used to find out soil texture that was loam-clay-loam that belongs to Hafizabad soil series (fine-loamy, mixed, hyperthermic, Typic Calciargids) (Khan, 1986). Soil pH and EC were $7.7 \pm 0.1$ and $2.13 \pm 0$. $\mathrm{dS} \mathrm{m}^{-1}$ that were determined by making use of Beckman 45 Modal, US a pH meter and VWR Conductivity Meter DIG2052 a EC meter, respectively. Organic content in soil was $0.67 \pm .2 \%$ calculated through Walkely-Block method, $\mathrm{N}$ was $78 \pm 2$ $\mathrm{mg} \mathrm{kg}^{-1}$ through Modified Kjeldahl Method, extractable-P was $55 \pm 4 \mathrm{mg} \mathrm{kg}^{-1}$ through Olsen's Method and available potassium was $573 \pm 15 \mathrm{mg} \mathrm{kg}^{-1}$ through Flame photometric during both years (Table 1).

Table 1. Physio-chemical characteristics of experimental site at College of Agriculture, University of Sargodha, Sargodha, Pakistan.

\begin{tabular}{lcccccc}
\hline Soil Characteristics & \multicolumn{3}{c}{ Spring-2018 } & \multicolumn{3}{c}{ Spring-2019 } \\
\cline { 2 - 7 } & $0-15 \mathrm{~cm}$ & $15-30 \mathrm{~cm}$ & Mean & $0-15 \mathrm{~cm}$ & $15-30 \mathrm{~cm}$ & Mean \\
\hline pH of soil & 7.8 & 7.5 & 7.6 & 7.7 & 7.6 & 7.6 \\
EC $\left(\mathrm{dS} \mathrm{m} \mathrm{m}^{-1}\right.$ ) & 2.19 & 2.08 & 2.13 & 2.22 & 2.09 & 2.15 \\
Organic matter (\%) & 0.72 & 0.58 & 0.65 & 0.73 & 0.60 & 0.67 \\
Total Nitrogen $\left(\mathrm{mg} \mathrm{kg}^{-1}\right)$ & 81 & 78 & 80 & 80 & 76 & 78 \\
Available Phosphorus $\left(\mathrm{mg} \mathrm{kg}^{-1}\right)$ & 63 & 47 & 55 & 66 & 51 & 59 \\
Available Potassium $\left(\mathrm{mg} \mathrm{kg}^{-1}\right)$ & 210 & 235 & 223 & 235 & 240 & 238 \\
\hline Texture & \multicolumn{5}{c}{ Sandy clay loam } & \multicolumn{4}{c}{ Sandy loam } \\
\hline
\end{tabular}


Climatic feature of district Sargodha is subtropical to semiarid having $400 \pm 5 \mathrm{~mm}$ annual rainfall. Monsoon seasons started in July and ended in September which received $70 \%$ of the total rainfall (Source: AgroMetrological Lab, University of Sargodha). Mean minimum temperature were about $10^{\circ} \mathrm{C}$ in winter season. Figure 1 depicted the concise data of weather during 2018 and 2019. In 2019 the mean maximum temperature at plant tillering, stem elongation, growth and juice quality during May, June, July, August and September were $10 \%, 20 \%, 21 \%, 12 \%$ and $13 \%$ higher than mean maximum temperature in 2018 respectively. In 2019 the mean minimum temperature during May, June, July, August, September, October and November were $10 \%$, $18 \%, 18 \%, 9 \%, 14 \%, 24 \%$ and $33 \%$ higher than mean minimum temperature in 2018 respectively. Moreover, during 2019, the mean temperature was higher from May and then steady higher on the way to maturity. Mean monthly rainfall was lower during $2019(400.5 \mathrm{~mm})$ as compared to 2018 (894.7 mm). Maximum rainfall was got during the months of April (269.9 mm), June (150.9 mm) and July (200.2 $\mathrm{mm}$ ) during 2018. Mean relative humidity was 41\% lower during 2019 than 2018.

\section{Experimental design and treatments}

Experimental treatments were arranged in a randomized complete block design with three replicates and $9 \mathrm{~m} \times 36$ $\mathrm{m}$ net plot size of each treatment during 2018 and 2019. Sugarcane (cv. YT-55) was sown $400 \mathrm{~kg} \mathrm{ha}^{-1}$ for budchips planting and maintaining row-to-row distance $75 \mathrm{~cm}$ and plant-to-plant distance $45 \mathrm{~cm}$ for budchips manual planting. Both years, experiment consist of nine treatments viz., $\mathrm{CaCl} 20 \%$ + NAA 0 ppm (control), $\mathrm{CaCl}_{2}$ $0 \%+\mathrm{NAA} 1 \mathrm{ppm}, \mathrm{CaCl}_{2} 0 \%+\mathrm{NAA} 2 \mathrm{ppm}, \mathrm{CaCl}_{2} 1 \%+\mathrm{NAA}$ $0 \mathrm{ppm}, \mathrm{CaCl}_{2} 1 \%+\mathrm{NAA} 1 \mathrm{ppm}, \mathrm{CaCl}_{2} 1 \%+\mathrm{NAA} 2 \mathrm{ppm}$, $\mathrm{CaCl}_{2} 2 \%+\mathrm{NAA} 0 \mathrm{ppm}, \mathrm{CaCl}_{2} 2 \%+\mathrm{NAA} 1 \mathrm{ppm}, \mathrm{CaCl}_{2} 2 \%$ + NAA 2 ppm on growth and production under the agroecological conditions of Sargodha.

\section{Preparation of the budchips}

Budchips were obtained from canes which was healthy and free from disease and pest. Sugarcane budchips were scooped by using Budchip/Budnode Scooping Machine of Sugarcane (Figure 1 Patent Number 143641) designed and fabricated by Dr. Abdul Rehman (Department of Agronomy, College of Agriculture, University of Sargodha, Pakistan); Dr. Muhammad Rafi Qamar (Department of Agronomy, College of Agriculture, University of Sargodha, Pakistan) and Dr. Atique-ur-Rehman (Department of Agronomy, Bahauddin Zakariya University, Multan,
Pakistan). Before planting, different concentrations of both $\mathrm{CaCl}_{2}$ and NAA chemicals were prepared respective to their treatments at room temperature $\left(25 \pm 1^{\circ} \mathrm{C}\right)$. Budchips were then soaked in respective solution for 2 hours then dipped into fungicide (Thiophenate methyle) @ $1.5 \mathrm{~g}$ /liter of water for 3 to 4 minutes. Afterwards, these budchips were sacked and dumped for 6 days. Sprouted budchips were taken out from soil and planted in the field during 2018 and 2019.

\section{Crop husbandry}

During both years of study, deep ploughing was done through two cross chiesel ploughings and then levelling with laser land leveler. A presoaking irrigation was given before preparing the final seedbed. When field attained proper soil moisture contents, soil was cultivated with tractor driven ordinary cultivator 3 times followed by planking. After that, ridges were made through ridger by maintaining ridge to ridge distance of $75 \mathrm{~cm}$. A total 80 budchips per line sown manually at the depth of $3.8 \mathrm{~cm}$. Recommended N, P and K @ 120, 70 and $50 \mathrm{~kg} \mathrm{ha}^{-1}$ doses were incorporated thoroughly into the soil. Complete dose of phosphorus, potassium and 1/3rd of the nitrogen dose was applied just before the ridge making. Remaining $2 / 3$ rd dose of nitrogen was applied in two splits i.e. $1 / 3 \mathrm{rd}$ start of tillering and $1 / 3$ rd just before the earthing up. Budchips were grown till maturity than experiment was discontinued and sugarcane plants were immersed in water for 2 hours and then removed carefully on January, 2018 and 2019. During study period, recommended agronomic practices were used and crop was kept free from insect-pests by applying pesticide.

\section{Data collection}

Sprouting establishment percentage, growth, yield and yield traits and quality traits were noted during and after the harvesting of plants in 2018 and 2019. For sprouting establishment percentage, plants were selected in each experimental plots and monitored regularly and sprouting percentage was calculated by the formula:

Sprouting establishment (SP)

$$
=\frac{\text { Sprouted or germinated buds }}{\text { Total buds planted }} \times 100
$$

Leaf area of green laminae was recorded on leaf area meter (LI-3100, LI-COR, Lincoln, NE) for calculating leaf area index by the formula (Watson, 1952).

Leaf area index $($ LAI $)=\frac{\text { Leaf area }}{\text { Land area }}$ 
Leaf area duration (LAD) (Days), crop growth rate (CGR) ( $\mathrm{g} \mathrm{m}^{-2}$ day $^{-1}$ ) and net assimilation rate (NAR) $\left(\mathrm{g} \mathrm{m}^{-2}\right.$ day $\left.^{-1}\right)$ were calculated according to the formula of Hunt (1978) respectively.

Leaf area duration (LAD) (Days)

$$
=\frac{(\mathrm{LAI} 1+\mathrm{LAI} 2) \times(\mathrm{T} 2-\mathrm{T} 1)}{2}
$$

Crop growth rate $(\mathrm{CGR})=\frac{(\mathrm{W} 2+\mathrm{W} 1)}{(\mathrm{T} 2-\mathrm{T} 1)}$

Net assimilation rate $(\mathrm{NAR})=\frac{\mathrm{TDM}}{\mathrm{LAD}}$

Meter rod was used for the measurement of cane height (cm) at maturity. For this purpose, ten plants were selected randomly from each treatment, height was noted from base to leaf tip and then average was calculated. At the time of harvesting, 3 random samples of $4 \mathrm{~m}^{2}$ were selected from each treatment. Number of tillers were counted from each sample, average of all samples was calculated and then worked into tillers $\mathrm{m}^{-2}$. Cane diameter (cm) was recorded with the help of Vernier caliper (Truper, CALDI-6MP), ten plant were randomly selected from each treatment and their diameter was recorded from base, middle and top portion and then averaged. For stripped cane yield, each plot was cut, stripped and topped than weighed on floor balance in $\mathrm{kg}$ than worked into tha-1. Commercial cane sugar (CCS) was calculated by adopting procedure of Spancer and Meade (1963).

$\operatorname{CCS}(\%)=\frac{3}{2} \mathrm{P}\left(1-\frac{\mathrm{F}+5}{100}\right)-\frac{1}{2} \mathrm{~B}\left(1-\frac{\mathrm{F}+5}{100}\right)$

Sugar yield ( $\mathrm{t} \mathrm{ha}{ }^{-1}$ ) was calculated by the following formula:

Sugar yield $\left(\mathrm{t} \mathrm{ha}^{-1}\right)$

$$
=\frac{\text { Stripped cane yield }\left(\mathrm{t} \mathrm{ha}^{-1}\right) \mathrm{XCC} \%}{100}
$$

\section{Statistical procedure used}

Study treatments were followed the statistical planning of randomized complete block design. SAS software (Version 9.1; SAS Institute, Cary, NC, USA) (SAS Institute, 2008) was applied for analysis (ANOVA) and means of treatments were separated by applying DMRT at 0.05 level of significance (Steel et al., 1997). Data showed in tables are means of three replicates of each year 2018 and 2019. Correlation was applied by using Minitab (Version 12, State College, PA, USA) (Minitab, Inc. 1998) to evaluate the role of experimental parameters on obtained results. Graphs were created using SigmaPlot software (Systat Software, 2008).

\section{RESULTS}

Effect of plant growth promotors on sugarcane budchips growth traits

Significantly $(\mathrm{P} \leq 0.05)$ higher sprouting establishment percentage $(86 \%)$ was noted in budchips which were soaked in $\mathrm{CaCl}_{2} 2 \%$ + NAA 2 ppm during 2018 and 2019. While lower sprouting establishment percentage (53 and $55 \%$ ) was recorded when budchips was not treated (control) $\mathrm{CaCl}_{2} 0 \%$ + NAA 0 ppm (Table 2). Statistically higher $(\mathrm{P} \leq 0.05)$ LAI (11.98 and 11.96) was calculated in $\mathrm{CaCl}_{2} 2 \%+\mathrm{NAA} 2 \mathrm{ppm}$ treatment and significantly $(\mathrm{P} \leq 0.05)$ lower was recorded in $\mathrm{CaCl}_{2} 0 \%+\mathrm{NAA} 0 \mathrm{ppm}$ (4.60 and 4.59) (Table 2) during 2018 and 2019. Both years showed maximum $(\mathrm{P} \leq 0.05)$ leaf duration $(1272$ and 1293 days) when budchips were soaked in $\mathrm{CaCl}_{2} 2 \%$ + NAA 2 ppm while minimum $(\mathrm{P} \leq 0.05)$ duration (808 and 816 days) was recorded in $\mathrm{CaCl}_{2} 0 \%+$ NAA 0 ppm (Table 2). PGPs significantly $(\mathrm{P} \leq 0.05)$ improved CGR $(11.58$ and $11.02 \mathrm{~g} \mathrm{~m}^{-2}$ day $^{-1}$ ) and NAR (2.53 and $2.55 \mathrm{~g} \mathrm{~m}^{-2}$ day $^{-1}$ ) where budchips were treated with $\mathrm{CaCl}_{2} 2 \%+\mathrm{NAA} 2 \mathrm{ppm}$ while significantly minimum CGR (6.97 and $6.66 \mathrm{~g} \mathrm{~m}^{-2}$ day $^{-1}$ ) and NAR (2.32 and $2.31 \mathrm{~g} \mathrm{~m}^{-2}$ day $^{-1}$ ) were recorded in control ( $\mathrm{CaCl}_{2}$ 0\%+ NAA 0 ppm) during 2018 and 2019 respectively (Table 2).

Effect of plant growth promotors on sugarcane budchips yield and quality traits

Significantly $(\mathrm{P} \leq 0.05)$ taller cane height $(203.70$ and 224 $\mathrm{cm})$ was measured when budchips were treated with $\mathrm{CaCl}_{2} 2 \%+$ NAA 2 ppm and shorter cane height (113.65 and $136 \mathrm{~cm}$ ) was recorded in $\mathrm{CaCl}_{2} 0 \%+\mathrm{NAA} 0 \mathrm{ppm}$ during both years (Table 3). In 2018 and 2019, $\mathrm{CaCl}_{2} 2 \%$ + NAA 2 ppm treatment produced higher number of tillers $\mathrm{m}^{-2}\left(27\right.$ and $\left.26 \mathrm{~m}^{-2}\right)$ while lower number of tillers were counted in $\mathrm{CaCl}_{2} 0 \%+$ NAA 0 ppm (14 and $13 \mathrm{~m}^{-2}$ ) (Table 3). Significantly $(\mathrm{P} \leq 0.05)$ thicker girth $(2.97$ and $3.01 \mathrm{~cm}$ ) was measured when budchips were soaked in $\mathrm{CaCl}_{2} 2 \%+\mathrm{NAA} 2 \mathrm{ppm}$ treatment. Among all the treatments, thinner girth was recorded in $\mathrm{CaCl}_{2} 0 \%+\mathrm{NAA}$ 0 ppm (2.25 and $2.14 \mathrm{~cm}$ ) during both years (Table 3). Budchips soaked in $\mathrm{CaCl}_{2} 2 \%+$ NAA 2 ppm produced higher $(\mathrm{P} \leq 0.05)$ stripped cane yield $(101.98$ and $98.52 \mathrm{t}$ $\mathrm{ha}^{-1}$ ) and sugar yield (16.77 and $16.75 \mathrm{tha}^{-1}$ ) while lower $(\mathrm{P} \leq 0.05)$ stripped cane yield (58.80 and $\left.58.70 \mathrm{t} \mathrm{ha}^{-1}\right)$ and sugar yield (7.71 and $7.69 \mathrm{t} \mathrm{ha}^{-1}$ ) were produced in $\mathrm{CaCl}_{2}$ 0\% + NAA 0 ppm during 2018 and 2019 respectively (Table 3). Significantly $(\mathrm{P} \leq 0.05)$ higher commercial cane 
sugar was calculated in $\mathrm{CaCl}_{2} 2 \%$ + NAA 2 ppm (14.45 and $14.39 \%)$. Among all the treatments, significantly $(\mathrm{P} \leq 0.05)$ lower commercial cane sugar $(10.87$ and
10.77\%) was recorded in $\mathrm{CaCl}_{2} 0 \%+$ NAA 0 ppm during 2018 and 2019 respectively (Table 3).

Table 2. Evaluating the effect of plant growth promoters on growth of sugarcane propagated through budchips.

\begin{tabular}{|c|c|c|c|c|c|c|c|c|c|c|}
\hline \multirow[t]{2}{*}{ Treatments } & \multicolumn{2}{|c|}{$\begin{array}{c}\text { Sprouting } \\
\text { establishment } \\
(\%)\end{array}$} & \multicolumn{2}{|c|}{ Leaf area index } & \multicolumn{2}{|c|}{$\begin{array}{l}\text { Leaf area duration } \\
\text { (days) }\end{array}$} & \multicolumn{2}{|c|}{$\begin{array}{l}\text { Crop growth rate } \\
\left(\mathrm{g} \mathrm{m}^{-2} \text { day }^{-1}\right)\end{array}$} & \multicolumn{2}{|c|}{$\begin{array}{l}\text { Net assimilation } \\
\text { rate }\left(\mathrm{g} \mathrm{m}^{-2} \text { day }^{-1}\right)\end{array}$} \\
\hline & 2018 & 2019 & 2018 & 2019 & 2018 & 2019 & 2018 & 2019 & 2018 & 2019 \\
\hline $\begin{array}{l}\mathrm{CaCl}_{2} 0 \%+ \\
\text { NAA } 0 \mathrm{ppm}\end{array}$ & $53 \mathrm{f}$ & 55 e & $4.60 \mathrm{e}$ & $4.59 \mathrm{e}$ & $808 \mathrm{f}$ & $804 \mathrm{f}$ & $6.97 \mathrm{f}$ & $6.66 \mathrm{e}$ & $2.32 \mathrm{~d}$ & $2.31 \mathrm{c}$ \\
\hline $\begin{array}{l}\mathrm{CaCl}_{2} 0 \%+ \\
\text { NAA } 1 \mathrm{ppm}\end{array}$ & $67 \mathrm{~d}$ & $67 \mathrm{~cd}$ & $5.67 \mathrm{de}$ & $5.66 \mathrm{de}$ & $888 d$ & $876 \mathrm{c}-\mathrm{e}$ & $8.52 \mathrm{c}-\mathrm{e}$ & $8.02 \mathrm{~cd}$ & $2.38 \mathrm{~cd}$ & $2.35 \mathrm{bc}$ \\
\hline $\begin{array}{l}\mathrm{CaCl}_{2} 0 \%+ \\
\mathrm{NAA} 2 \mathrm{ppm}\end{array}$ & $73 \mathrm{bc}$ & $74 \mathrm{bc}$ & $6.38 \mathrm{~cd}$ & $6.37 \mathrm{~cd}$ & $872 \mathrm{de}$ & $867 \mathrm{de}$ & $9.44 \mathrm{bc}$ & $8.91 \mathrm{bc}$ & $2.45 \mathrm{a}-\mathrm{c}$ & $2.45 \mathrm{ab}$ \\
\hline $\begin{array}{l}\mathrm{CaCl}_{2} 1 \%+ \\
\text { NAA } 0 \mathrm{ppm}\end{array}$ & 59 ef & $60 \mathrm{de}$ & $5.46 \mathrm{de}$ & $5.45 \mathrm{de}$ & 822 ef & 816 ef & $8.03 \mathrm{de}$ & $7.52 \mathrm{de}$ & $2.48 \mathrm{ab}$ & $2.50 \mathrm{a}$ \\
\hline $\begin{array}{l}\mathrm{CaCl}_{2} 1 \%+ \\
\text { NAA } 1 \mathrm{ppm}\end{array}$ & $70 \mathrm{~cd}$ & $71 \mathrm{bc}$ & $8.02 \mathrm{c}$ & $8.00 \mathrm{c}$ & 947 c & $932 \mathrm{c}$ & $8.96 \mathrm{~b}-\mathrm{d}$ & $9.11 \mathrm{~b}$ & $2.44 \mathrm{a}-\mathrm{c}$ & $2.47 \mathrm{a}$ \\
\hline $\begin{array}{l}\mathrm{CaCl}_{2} 1 \%+ \\
\text { NAA } 2 \mathrm{ppm}\end{array}$ & $75 \mathrm{bc}$ & $75 \mathrm{~b}$ & $9.95 \mathrm{~b}$ & $9.93 \mathrm{~b}$ & $1095 \mathrm{~b}$ & $1077 \mathrm{~b}$ & $11.11 \mathrm{a}$ & $10.85 \mathrm{a}$ & $2.50 \mathrm{ab}$ & $2.50 \mathrm{a}$ \\
\hline $\begin{array}{l}\mathrm{CaCl}_{2} 2 \%+ \\
\text { NAA } 0 \mathrm{ppm}\end{array}$ & $61 \mathrm{e}$ & $61 \mathrm{de}$ & $6.59 \mathrm{~cd}$ & $6.57 \mathrm{~cd}$ & 873 de & 863 ef & 7.64 ef & $7.18 \mathrm{de}$ & $2.42 \mathrm{~b}-\mathrm{d}$ & $2.45 \mathrm{ab}$ \\
\hline $\begin{array}{l}\mathrm{CaCl}_{2} 2 \%+ \\
\text { NAA } 1 \mathrm{ppm}\end{array}$ & $78 \mathrm{~b}$ & $77 \mathrm{~b}$ & $7.55 \mathrm{c}$ & $7.54 \mathrm{c}$ & $915 \mathrm{~cd}$ & $925 \mathrm{~cd}$ & $9.51 \mathrm{~b}$ & $9.21 \mathrm{~b}$ & $2.50 \mathrm{ab}$ & $2.48 \mathrm{a}$ \\
\hline $\begin{array}{l}\mathrm{CaCl}_{2} 2 \%+ \\
\text { NAA } 2 \mathrm{ppm}\end{array}$ & $86 a$ & $86 \mathrm{a}$ & $11.98 \mathrm{a}$ & $11.96 \mathrm{a}$ & $1272 \mathrm{a}$ & $1293 a$ & $11.58 \mathrm{a}$ & $11.02 \mathrm{a}$ & $2.53 \mathrm{a}$ & $2.55 \mathrm{a}$ \\
\hline $\begin{array}{l}\text { LSD value } \\
\text { at } 5 \% \\
\text { probability } \\
\end{array}$ & 5.81 & 7.50 & 1.69 & 1.67 & 55.39 & 59.96 & 0.96 & 1.03 & 0.11 & 0.11 \\
\hline $\mathrm{CV}$ & 5.77 & 6.25 & 15.78 & 15.77 & 4.02 & 3.69 & 7.28 & 6.88 & 2.95 & 2.68 \\
\hline
\end{tabular}

Table 3. Evaluating the effect of plant growth promoters on yield of sugarcane propagated through budchips.

\begin{tabular}{|c|c|c|c|c|c|c|c|c|c|c|c|c|}
\hline \multirow[t]{2}{*}{ Treatment } & \multicolumn{2}{|c|}{ Cane height (cm) } & \multicolumn{2}{|c|}{$\begin{array}{l}\text { Number of } \\
\text { tillers } \mathrm{m}^{-2}\end{array}$} & \multicolumn{2}{|c|}{$\begin{array}{l}\text { Cane diameter } \\
(\mathrm{cm})\end{array}$} & \multicolumn{2}{|c|}{$\begin{array}{l}\text { Stripped cane } \\
\text { yield }\left(\mathrm{t} \mathrm{ha}^{-1}\right)\end{array}$} & \multicolumn{2}{|c|}{$\begin{array}{c}\text { Commercial } \\
\text { cane sugar (\%) }\end{array}$} & \multicolumn{2}{|c|}{$\begin{array}{c}\text { Sugar yield (t ha- } \\
1 \text { ) }\end{array}$} \\
\hline & 2018 & 2019 & 2018 & 2019 & 2018 & 2019 & 2018 & 2019 & 2018 & 2019 & 2018 & 2019 \\
\hline $\begin{array}{l}\mathrm{CaCl}_{2} 0 \%+ \\
\text { NAA } 0 \text { ppm }\end{array}$ & $113.6 \mathrm{f}$ & $136 \mathrm{~g}$ & $14 \mathrm{~d}$ & $13 \mathrm{e}$ & $2.25 \mathrm{~d}$ & $2.14 \mathrm{c}$ & $58.8 \mathrm{i}$ & $58.7 \mathrm{~h}$ & $10.8 \mathrm{~b}$ & $10.7 \mathrm{~b}$ & $7.71 \mathrm{e}$ & $7.69 \mathrm{e}$ \\
\hline $\begin{array}{l}\mathrm{CaCl}_{2} 0 \%+ \\
\mathrm{NAA} 1 \mathrm{ppm}\end{array}$ & $137.9 \mathrm{de}$ & $162 \mathrm{f}$ & $17 \mathrm{~cd}$ & $15 \mathrm{de}$ & $2.41 \mathrm{~cd}$ & $2.40 \mathrm{bc}$ & $61.7 \mathrm{~g}$ & $61.5 \mathrm{~g}$ & $\begin{array}{c}12.9 \\
\mathrm{ab}\end{array}$ & $\begin{array}{c}13.4 \\
\mathrm{ab}\end{array}$ & $9.22 \mathrm{de}$ & $9.20 \mathrm{de}$ \\
\hline $\begin{array}{l}\mathrm{CaCl}_{2} 0 \%+ \\
\mathrm{NAA} 2 \mathrm{ppm}\end{array}$ & $155.7 \mathrm{bc}$ & $181 \mathrm{e}$ & $20 \mathrm{bc}$ & $19 \mathrm{bc}$ & $2.49 \mathrm{~b}-\mathrm{d}$ & $2.47 \mathrm{~b}$ & $71.0 \mathrm{e}$ & $65.4 \mathrm{e}$ & $\begin{array}{c}13.4 \\
\mathrm{ab}\end{array}$ & $\begin{array}{l}14.2 \\
\mathrm{ab}\end{array}$ & $10.9 \mathrm{~cd}$ & $10.9 \mathrm{~cd}$ \\
\hline $\begin{array}{l}\mathrm{CaCl}_{2} 1 \%+ \\
\mathrm{NAA} 0 \mathrm{ppm}\end{array}$ & 130.2 ef & $158 \mathrm{f}$ & $16 \mathrm{~d}$ & $15 \mathrm{de}$ & $2.34 \mathrm{~cd}$ & $2.40 \mathrm{bc}$ & $59.9 \mathrm{~h}$ & $59.9 \mathrm{~h}$ & $\begin{array}{l}12.3 \\
\mathrm{ab}\end{array}$ & $\begin{array}{c}11.9 \\
\mathrm{ab}\end{array}$ & $8.41 \mathrm{de}$ & $8.39 \mathrm{de}$ \\
\hline $\begin{array}{l}\mathrm{CaCl}_{2} 1 \%+ \\
\mathrm{NAA} 1 \mathrm{ppm}\end{array}$ & $169.5 \mathrm{~b}$ & $198 \mathrm{c}$ & $22 \mathrm{~b}$ & $21 \mathrm{~b}$ & $2.74 \mathrm{ab}$ & $2.44 \mathrm{~b}$ & $86.5 \mathrm{c}$ & $70.5 \mathrm{c}$ & $\begin{array}{l}12.0 \\
\mathrm{ab}\end{array}$ & $\begin{array}{c}13.2 \\
\mathrm{ab}\end{array}$ & $12.1 \mathrm{bc}$ & $12.1 \mathrm{bc}$ \\
\hline $\begin{array}{l}\mathrm{CaCl}_{2} 1 \%+ \\
\mathrm{NAA} 2 \mathrm{ppm}\end{array}$ & $186.7 \mathrm{a}$ & $207 \mathrm{~b}$ & $23 \mathrm{~b}$ & $22 \mathrm{~b}$ & $2.88 \mathrm{a}$ & $2.79 \mathrm{a}$ & $91.2 \mathrm{~b}$ & $83.2 \mathrm{~b}$ & $\begin{array}{c}13.1 \\
\mathrm{ab}\end{array}$ & $\begin{array}{c}14.3 \\
\mathrm{ab}\end{array}$ & $13.84 \mathrm{~b}$ & $13.82 \mathrm{~b}$ \\
\hline
\end{tabular}




\begin{tabular}{|c|c|c|c|c|c|c|c|c|c|c|c|c|}
\hline $\begin{array}{l}\mathrm{CaCl}_{2} 2 \%+ \\
\text { NAA } 0 \text { ppm }\end{array}$ & $151.2 \mathrm{~cd}$ & 177 e & $22 \mathrm{~b}$ & $17 \mathrm{~cd}$ & $2.41 \mathrm{~cd}$ & $2.36 \mathrm{bc}$ & $64.2 \mathrm{f}$ & $63.4 \mathrm{f}$ & $\begin{array}{c}12.1 \\
\mathrm{ab}\end{array}$ & $\begin{array}{c}12.0 \\
\mathrm{ab}\end{array}$ & $9.10 \mathrm{de}$ & $9.07 \mathrm{de}$ \\
\hline $\begin{array}{l}\mathrm{CaCl}_{2} 2 \%+ \\
\mathrm{NAA} 1 \mathrm{ppm}\end{array}$ & $164.4 \mathrm{bc}$ & $191 \mathrm{~d}$ & $22 \mathrm{~b}$ & $22 \mathrm{~b}$ & $2.54 \mathrm{bc}$ & $2.85 \mathrm{a}$ & $80.8 \mathrm{~d}$ & $68.9 \mathrm{~d}$ & $\begin{array}{c}14.2 \\
\mathrm{ab}\end{array}$ & $\begin{array}{c}13.9 \\
a b\end{array}$ & $13.0 \mathrm{bc}$ & $13.0 \mathrm{bc}$ \\
\hline $\begin{array}{l}\mathrm{CaCl}_{2} 2 \%+ \\
\mathrm{NAA} 2 \mathrm{ppm}\end{array}$ & 203.7 a & $224 \mathrm{a}$ & $27 \mathrm{a}$ & $26 \mathrm{a}$ & $2.97 \mathrm{a}$ & $3.01 \mathrm{a}$ & $\begin{array}{c}101.9 \\
\mathrm{a}\end{array}$ & $98.5 \mathrm{a}$ & $14.4 \mathrm{a}$ & $14.3 \mathrm{a}$ & $16.7 \mathrm{a}$ & $16.7 \mathrm{a}$ \\
\hline $\begin{array}{l}\text { LSD value } \\
\text { at } 5 \% \\
\text { probability }\end{array}$ & 16.96 & 5.14 & 3.43 & 3.43 & 0.28 & 0.28 & 1.14 & 1.48 & 2.43 & 2.41 & 2.59 & 2.57 \\
\hline $\mathrm{CV}$ & 7.40 & 1.65 & 9.84 & 10.59 & 7.78 & 6.54 & 0.78 & 1.22 & 15.57 & 15.20 & 15.75 & 15.72 \\
\hline
\end{tabular}

Values sharing same letter (s) in a column did not differ significantly at 5\% probability, CV = Coefficient of variation

Table 4. Pearson's coefficient correlation among sugarcane budchip parameters 2018. NS insignificant; * - significant at $\mathrm{P} \leq 0.05$; $^{* *}$ - significant at $\mathrm{P} \leq 0.01$.

\begin{tabular}{|c|c|c|c|c|c|c|c|c|c|c|c|}
\hline & SE & LAI & LAD & CGR & NAR & $\mathrm{CH}$ & NT & $\mathrm{CD}$ & STY & $\mathrm{CCS}$ & SY \\
\hline SE & 1 & & & & & & & & & & \\
\hline LAI & $0.85^{* *}$ & 1 & & & & & & & & & \\
\hline LAD & $0.82^{* *}$ & $0.97^{* *}$ & 1 & & & & & & & & \\
\hline CGR & $0.93^{* *}$ & $0.92^{* *}$ & $0.90^{* *}$ & 1 & & & & & & & \\
\hline NAR & $0.78^{*}$ & $0.76^{*}$ & $0.66^{*}$ & $0.80^{*}$ & 1 & & & & & & \\
\hline $\mathrm{CH}$ & $0.91^{* *}$ & $0.97^{* *}$ & $0.91^{* *}$ & $0.93^{* *}$ & $0.80^{* *}$ & 1 & & & & & \\
\hline NT & $0.85^{* *}$ & $0.92^{* *}$ & $0.84^{* *}$ & $0.81^{* *}$ & $0.75^{*}$ & $0.96^{* *}$ & 1 & & & & \\
\hline CD & $0.84^{* *}$ & $0.97^{* *}$ & $0.93^{* *}$ & $0.92^{* *}$ & $0.72^{*}$ & $0.96^{* *}$ & $0.88^{* *}$ & 1 & & & \\
\hline STY & $0.88^{* *}$ & $0.96^{* *}$ & $0.91^{* *}$ & $0.91^{* *}$ & $0.73^{*}$ & $0.96^{* *}$ & $0.89^{* *}$ & $0.98^{* *}$ & 1 & & \\
\hline CCS & $0.82^{* *}$ & $0.60^{\mathrm{NS}}$ & $0.62^{\mathrm{NS}}$ & $0.69^{*}$ & $0.40^{\mathrm{NS}}$ & $0.63^{\mathrm{NS}}$ & $0.62^{\mathrm{NS}}$ & $0.54^{\mathrm{NS}}$ & $0.63^{\mathrm{NS}}$ & 1 & \\
\hline SY & $0.95^{* *}$ & $0.96^{* *}$ & $0.92^{* *}$ & $0.95^{* *}$ & $0.78^{*}$ & $0.96^{* *}$ & $0.90^{* *}$ & $0.94^{* *}$ & $0.98^{* *}$ & $0.74^{*}$ & 1 \\
\hline
\end{tabular}

Sprouting establishment $=$ SE; Leaf area index $=$ LAI; Leaf area duration (Days) $=$ LAD; Crop growth rate $\left(\mathrm{g} \mathrm{m}^{-2}\right.$ day $\left.^{-1}\right)=$ CGR; Net assimilation rate $\left(\mathrm{g} \mathrm{m}^{-2}\right.$ day $\left.\mathrm{y}^{-1}\right)=N A R$; Cane height $(\mathrm{cm})=C H ;$ Number of tillers $\left(\mathrm{m}^{-2}\right)=N T$; Cane diameter $(\mathrm{cm})=$ $C D$; Stripped cane yield $\left(t h a^{-1}\right)=S T Y ;$ Commercial cane sugar (\%) $=C C S ;$ Sugar yield $\left(t h a^{-1}\right)=S Y$.

Table 5. Pearson's coefficient correlation among sugarcane budchip parameters 2019. NS insignificant; * - significant at $\mathrm{P} \leq 0.05 ;{ }^{* *}$ - significant at $\mathrm{P} \leq 0.01$.

\begin{tabular}{|c|c|c|c|c|c|c|c|c|c|c|c|}
\hline & SE & LAI & LAD & CGR & NAR & $\mathrm{CH}$ & NT & $\mathrm{CD}$ & STY & CCS & SY \\
\hline SE & 1 & & & & & & & & & & \\
\hline LAI & $0.86^{* *}$ & 1 & & & & & & & & & \\
\hline LAD & $0.83^{* *}$ & $0.97^{* *}$ & 1 & & & & & & & & \\
\hline CGR & $0.93^{* *}$ & $0.92^{* *}$ & $0.88^{* *}$ & 1 & & & & & & & \\
\hline NAR & $0.67^{*}$ & $0.75^{*}$ & $0.65^{*}$ & $0.71^{*}$ & 1 & & & & & & \\
\hline $\mathrm{CH}$ & $0.90^{* *}$ & $0.95^{* *}$ & $0.86^{* *}$ & $0.92^{* *}$ & $0.81^{* *}$ & 1 & & & & & \\
\hline NT & $0.94^{* *}$ & $0.94^{* *}$ & $0.87^{* *}$ & $0.92^{* *}$ & $0.79^{*}$ & $0.97^{* *}$ & 1 & & & & \\
\hline CD & $0.92^{* *}$ & $0.88^{* *}$ & $0.85^{* *}$ & $0.90^{* *}$ & $0.77^{*}$ & $0.87^{* *}$ & $0.91^{* *}$ & 1 & & & \\
\hline STY & $0.84^{* *}$ & $0.98^{* *}$ & $0.99^{* *}$ & $0.90^{* *}$ & $0.69^{*}$ & $0.89^{* *}$ & $0.89^{* *}$ & $0.86^{* *}$ & 1 & & \\
\hline CCS & $0.86^{* *}$ & $0.66^{*}$ & $0.64^{\mathrm{NS}}$ & $0.81^{* *}$ & $0.29^{\mathrm{NS}}$ & $0.72^{*}$ & $0.74^{*}$ & $0.69^{*}$ & $0.65^{*}$ & 1 & \\
\hline SY & $0.95^{* *}$ & $0.96^{* *}$ & $0.93^{* *}$ & $0.96^{* *}$ & $0.73^{*}$ & $0.95^{* *}$ & $0.98^{* *}$ & $0.94^{* *}$ & $0.94^{* *}$ & $0.78^{*}$ & 1 \\
\hline
\end{tabular}

Sprouting establishment $=\mathrm{SE}$; Leaf area index $=\mathrm{LAI}$; Leaf area duration (Days) $=$ LAD; Crop growth rate $\left(\mathrm{g} \mathrm{m}^{-2}\right.$ day $\left.^{-1}\right)=$ CGR; Net assimilation rate $\left(\mathrm{g} \mathrm{m}^{-2}\right.$ day $\left.^{-1}\right)=\mathrm{NAR}$; Cane height $(\mathrm{cm})=\mathrm{CH}$; Number of tillers $\left(\mathrm{m}^{-2}\right)=\mathrm{NT}$; Cane diameter $(\mathrm{cm})$ $=\mathrm{CD}$; Stripped cane yield $\left(\mathrm{t} \mathrm{ha}^{-1}\right)=\mathrm{STY}$; Commercial cane sugar $(\%)=\mathrm{CCS}$; Sugar yield $\left(\mathrm{t} \mathrm{ha}^{-1}\right)=\mathrm{SY}$. 


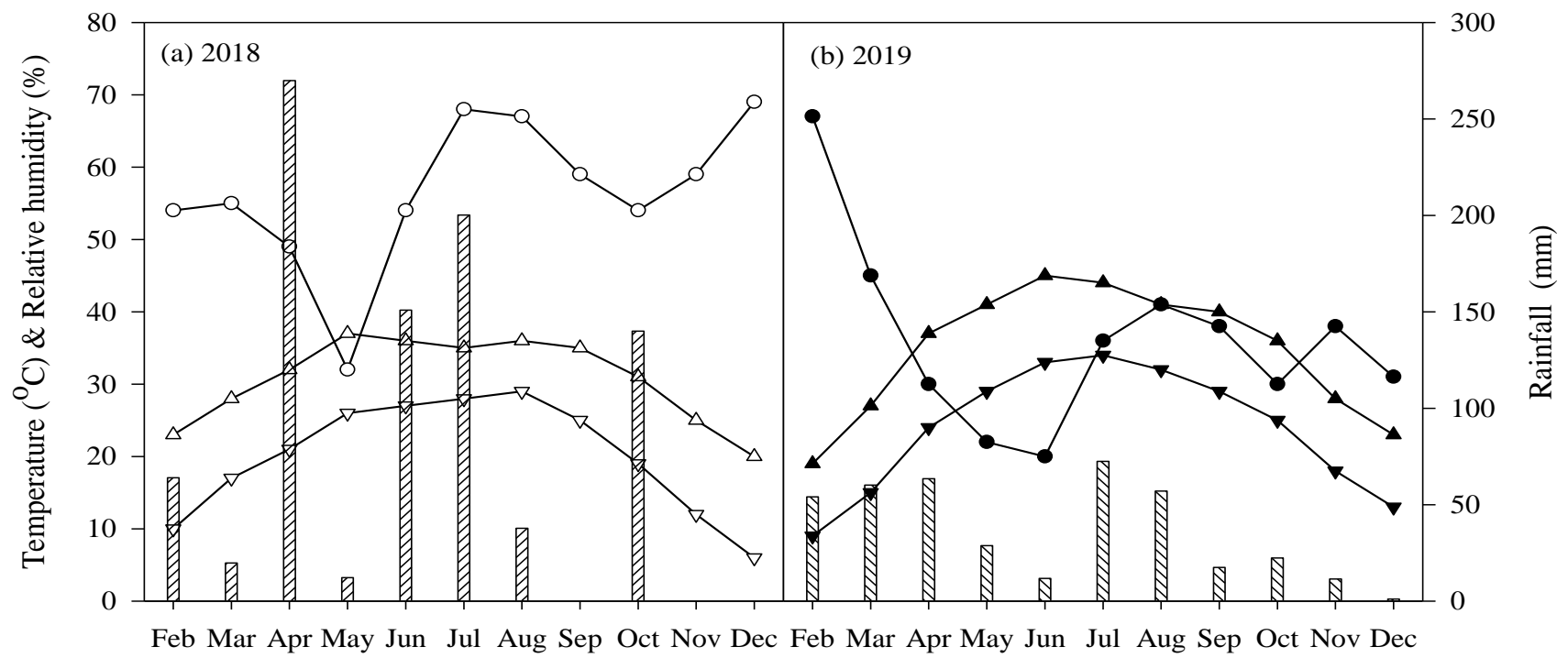

Months

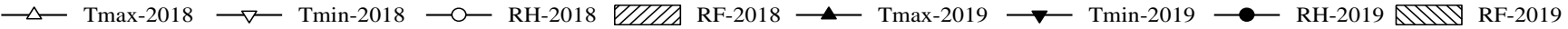

Figure 1. Climatic conditions prevailed during study at College of Agriculture, University of Sargodha, Sargodha, Pakistan in 2018 and 2019.

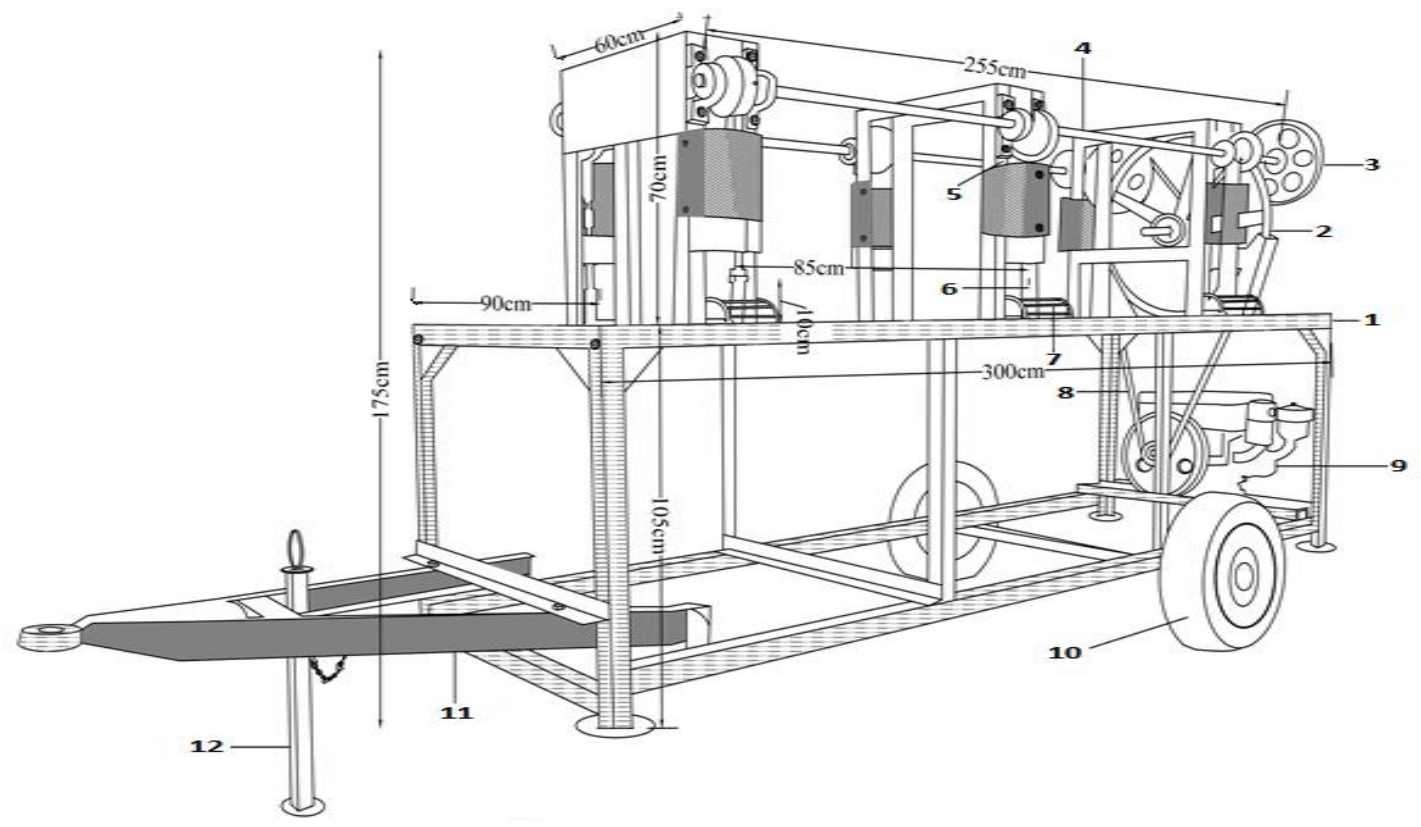

Figure 2. Budchip/budnode scooping machine (Basic parts; 1- Main frame; 2- Large pulley; 3-Small pulley; 4Shaft/beam; 5- Cam; 6- Cutter; 7- Protecting cover; 8- Belt; 9- Peter engine; 10- Wheel; 11- V-shaped hook; 12- Stand) (Intellectual Property Organization Karachi Pakistan; The Patents (Amendments) Act, 2016; (Patent Number 143641). 


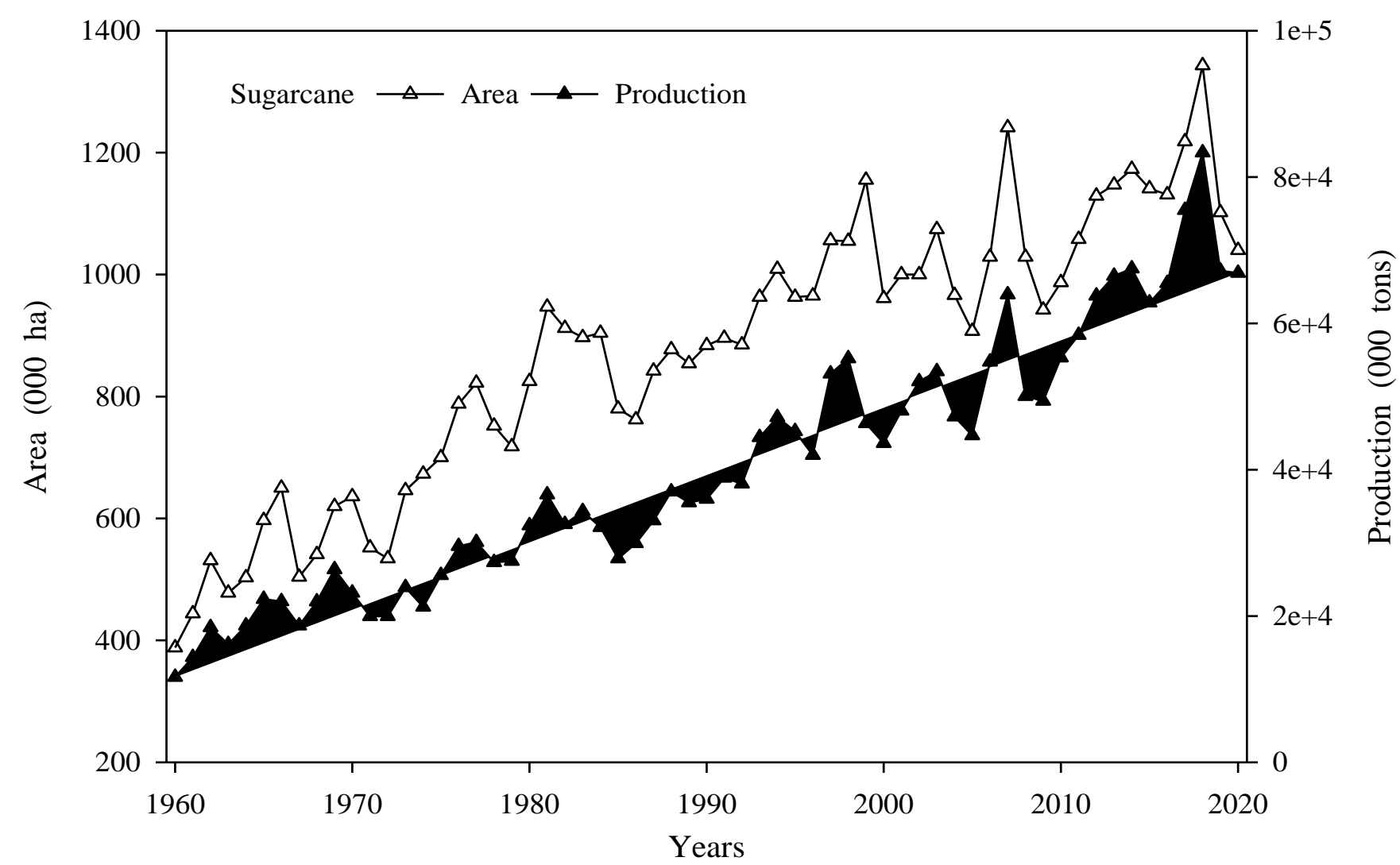

Figure 3. Historical area and production of sugarcane crop from 1960 to 2020 in Pakistan (Source: Modified and adapted from Ahmad et al., 2018).

\section{DISCUSSION}

Plant growth promotors have significant influence on growth, yield and quality of sugarcane budchip (Table 2 and 3) during 2018 and 2019. Regarding growth parameters, significantly $(\mathrm{P} \leq 0.05)$ higher sprouting percentage (Table 2) in all PGPs treatments over control might be due to increase in concentration of glucose, fructose and acid invertase enzymes in sprouting sugarcane buds. Our study findings corroborate the findings of Rehman et al. (2021) who concluded that budchip soaking in NAA and $\mathrm{CaCl}_{2}$ enhanced the sprouting percentage of sugarcane budchips. PGPs in combined form significantly improve the sprouting establishment (Table 2) might be to enhance the activity of ATPase (Jain et al., 2009). Moreover, PGPs enhanced the potential of cytokinin that leads to stimulate cotyledon growth (White and Broadley, 2003). Higher leaf area index in treatment of $\mathrm{CaCl}_{2} 2 \%+\mathrm{NAA} 2 \mathrm{ppm}$ (Table 2) might be possible due to availability of high moisture content that leads to conversion of carbohydrates into reducing sugar which favored plant growth (Rehman et al., 2021). Significant ( $\mathrm{P} \leq 0.05)$ improvement was recorded in crop growth rate and net assimilation rate when sugarcane budchips were soaked in $2 \% \mathrm{CaCl}_{2}$ and 2 ppm NAA solution (Table 2). Rehman et al. (2021) who conformed our findings by revealing that sugarcane budchips treated with $\mathrm{CaCl}_{2}$ and NAA showed significant increase in crop growth such as CGR and NAR as compared to control. Budchips soaked in plant growth promoter's treatment $\mathrm{CaCl}_{2} 2 \%+\mathrm{NAA} 2$ ppm leads to significant $(\mathrm{P} \leq 0.05)$ improvement in yield and quality traits during 2018 and 2019 (Table 3). Plant growth promoters might enhance the activity of acid invertase and ATPase, which convert the sugar into reducing form, which is direct source of food that leaded to taller plants than control (Table 3). In our experiment, budchips soaking in PGPs improved sprouting establishment (Table 3), which might increase reducing sugars as a results of acid invertase enzymes in sprouted buds that leads to promote cell division, growth, differentiation and production of sucrose in budding meristem of sugarcane (Abd El-Lattief and Bekheet, 2012 and Rehman et al., 2021). The result reveals that soaking of budchip with PGPs can be useful in improving 
sprouting establishment that resulted in more stem diameter, tillers and stripped cane yield (Table 3). The significant improvement in yield traits in PGPs treatment (Rehman et al., 2021) might be due to increase in ATPase and acid invertase activity. Our study sustained the results of Jain et al. (2007) who confirmed that soaking of budchip in $\mathrm{CaCl}_{2} 2 \%+$ NAA 2 ppm (PGPs) converted sucrose into glucose which is rapidly mobilized and utilized by the growing point of the sugarcane bud that leads to improve in yield and quality traits (Table 3). Furthermore, our findings are similar to Rehman et al. (2021), they reported that application of PGPs at initial stage significantly improved the in growth and yield characteristics of sugarcane budchip (Table 2 and 3). Moreover, NAA is a synthetic auxin which play an important role in different growth progressions (Zhao, 2010) and $\mathrm{CaCl}_{2}$ stimulates root initiation and growth (Hepler, 2005).

Correlation coefficient of sugarcane budchips during 2018 and 2019 are presented in the Table 4 and 5. During 2018 and 2019, correlation in different variables clearly directed that seedling establishment showed highly significant $(\mathrm{P} \leq 0.05)$ positive correlation with LAI, LAD, CGR, NAR, cane height, tillers, cane diameter, stripped yield, CCS and sugar yield (Table 4 and 5). During 2018 and 2019, stripped cane yield depicted highly significant $(P \leq 0.05)$ positive correlation with seedling establishment, LAI, LAD, CGR, NAR, cane height, tillers, cane diameter, CCS and sugar yield while non-significant correlation with CCS only in 2018 (Table 4 and 5). In 2018, commercial cane sugar had non-significant $(\mathrm{P} \leq 0.05)$ correlation with LAI, LAD, net assimilation rate, cane height, tillers, cane diameter and stripped yield (Table 4), while in 2019, only non-significant $(\mathrm{P} \leq 0.05)$ correlated with LAD and NAR (Table 5).

\section{CONCLUSION}

From the results of both years' field study, it is concluded that soaking treatment of sugarcane budchips with plant growth promoters @ 2\% $\mathrm{CaCl}_{2}$ and 2 ppm NAA solution improves their growth, yield and quality parameters. Hence, it improved the performance of novel budchip technology with a great scope for better seed handling at grower's level and improve sugarcane productivity. Therefore, on the basis of the above study; it is recommended to the sugarcane farmers who interested to adopt this new planting technique, soaked budchip in solution of $\mathrm{CaCl}_{2}$ and NAA for obtaining higher sprouting establishment and production.

\section{ACKNOWLEDGEMENTS}

The authors of this manuscript are thankful to the Higher Education Commission (HEC) in Islamabad, Pakistan for financial support during the duration of study.

\section{REFERENCES}

Abd El-Lattief, E. A., \& Bekheet, M. A. 2012. Quantitative and qualitative attributes of three sugarcane varieties as influenced by foliar spray of some growth regulators under upper Egypt conditions. Sugar Tech, 14(4), 345-350.

Ahmad, S., Atique-ur-Rehman, Ejaz, M., Fatima, Z., Kan, M., \& Ahmad, M. 2018. Agricultural land-use change of major field crops in Pakistan (1961-2014). Science, Technology and Development, 37(3): 113-121.

Aslam, M., Sultana, B. \& Ali, S. 2013. Alteration in antioxidant potential of Spinacia oleracea L. in response to selected plant growth regulators. Pakistan Journal of Agricultural Sciences, 50(4), 699-706.

Davies, P. J. 2010. The plant hormones: their nature, occurrence, and functions. Springer, Dordrecht, pp. 1-15.

Galal, M. O. A. 2016. A new technique for planting sugarcane in Egypt. Institute of Integrative Omics and Applied Biotechnology Journal, 7(4), 15-21.

Hepler, P. K. 2005. Calcium: a central regulator of plant growth and development. The Plant Cell, 17(8), 2142-2155.

Hunt, R. 1978. Plant growth analysis. Edward Arnold U. K: 26-38.

Iqbal, M., Ashraf, M., Jamil, A. \& Ur-Rehman, S. 2006. Does seed priming induce changes in the levels of some endogenous plant hormones in hexaploid wheat plants under salt stress? Journal of Integrative Plant Biology, 48(2), 181-189.

Jain R, Shrivastava A. K., Solomon S. \& Yadav R. L. 2007. Low temperature stress-induced biochemical changes in stubble bud sprouting in sugarcane (Saccharum sp. hybrid). Plant Growth Regulator. 53: 17-23.

Jain, R., Solomon, S., Shrivastava, A. K. \& Chandra, A. 2010. Sugarcane bud chips: a promising seed material. Sugar Tech, 12(1), 67-69.

Jain, R., Solomon, S., Shrivastava, A. K. \& Lal, P. 2009. Nutrient application improves stubble bud sprouting under low temperature conditions in 
sugarcane. Sugar Tech, 11(1), 83-85.

Khan, M.N., Hussain, M., Abbas, G., Fatima, Z., Iqbal, P., Khan, A., Zakir, I., Ahmed, M., Mateen, A., Khan, M.A., \& Ahmad, S. 2020. Improving resource use efficiencies of sugarcane at farmer field under arid environment. International Journal of Agriculture \& Biology, 24(5), 1279-1285.

Khan G. S. 1986. Need for international crosschecking and correlation in soil analysis for international classification systems. In: Proceedings of the Twelfth International Forum on Soil Taxonomy and Agro-Technology Transfer: Soil Survey of Pakistan. Volume 1. Lahore: Director General, Soil Survey of Pakistan. pp: 276-293.

Minitab Inc. 1998. MINITAB release 12 for windows. Brooks/Cole, Pacific Grove.

Mohanty, M. \& Nayak, P. K. 2011. Economizing seed cane quantity by reducing sett size and bud number with sett treatment in sugarcane cultivation. Indian Journal of Sugarcane Technology, 26(2), 59-60.

Mohanty, S. K. \& Sahoo, N. C. 2000. Effect of soaking period, seed size and growth regulators on imbibition and germination of seeds of some field crops. Orissa J. Agric. Res. Pub. CAB Abstracts.

Padilla-Fernandez, M. D., Gonzales, J. P., \& Mamauag, M. J. 2020. Improving farm practices and productivity through training needs assessment of sugarcane farmers: the case of Davao Mill District, the Philippines. International Journal of Agricultural Extension, 8(2), 113-129.

Patil, R. S., Patil, D. B., Patil, D. M., Shashikant, B. \& Khandagale 2018. Cam operated sugarcane bud cutting machine. International Research Journal of Engineering Technology, 5(10), 140-147.

Patnaik, J. R., Singh, S. N., Sarangi, D. \& Nayak, P. K. 2017. Assessing potentiality of bud chip technology on sugarcane productivity, profitability and sustainability in real farming situations under south east coastal plain zone of Odisha, India. Sugar Tech, 19(4), 373-377.
Paull, J. G. Cartwright, B. \& Rathjen, A. J. 1998. Responses of wheat and barley genotypes to toxic concentrations of soil boron. Euphytica. 39: 137-144.

Prakash, M., \& Ganesan, J. 2000. Effect of plant growth regulators and micronutrients on certain growth analysis parameters in sesame. Sesame and Safflower Newsletter, (15), 48-58.

Rehman, A., Hassan, F. Qamar, R. \& Rehman, A. U. 2021. Application of plant growth promoters on sugarcane (Saccharum officinarum L.) budchip under subtropical conditions. Asian Journal of Agricultural Biology. 2: 1-10.

Ryan, J., Estfan, G. \& Rashid, A. 2001. Soil and Plant Analysis Laboratory Manual. 2nd edition. ICARDA, Aleppo, Syria. 172 p.

SAS Institute. 2008. SAS/STAT 9.1 User's Guide: The REG Procedure (Book Excerpt). Cary, NC: SAS Institute

Shoo, H. K. (2014). Effect of planting methods on yield and economics of sugarcane. National symposium on management option farm productivity and livelihood security under changing climate, Odisha, ISA, pp. 221-222.

Spancer, G. L., \& Meade, G. P. 1963. Cane sugar hand book. $9^{\text {th }}$ Ed. G.P. Meade. John Wiley and Sons, Inc. New York. pp: 17.

Steel, R. G. D., Torrie, J. H. Dicky, D. A. 1997. Principles and Procedures of Statistics: A Biometric Approach. 3rd ed. New York: McGraw-Hill. Pp 178-198.

Systat Software Inc. 2008. SigmaPlot for Windows (development and testing procedures). Version 11.0. Triestram and Partner GmbH, Bochum, Germany.

Watson, D. J. 1952. The physiological basis of variation in yield. In Advances in agronomy (Vol. 4, pp. 101145). Academic Press.

White, P.J., \& Broadley, M. R. 2003. Calcium in plants. Annals of Botany, 92(0), 1-25.

Zhao, Y. 2010. Auxin biosynthesis and its role in plant development. Annual review of plant biology, 61, 49-64. 